\title{
A finite thickness band method for ductile fracture analysis
}

\author{
A.E. Huespe ${ }^{a}$, , A. Needleman ${ }^{b}$, J. Oliver ${ }^{\mathrm{c}}$ and P.J. Sánchez ${ }^{\underline{a}}$ \\ ${ }^{a}$ CIMEC-INTEC-UNL-CONICET, Güemes 3450, 3000 Santa Fe, Argentina \\ ${ }^{b}$ Division of Engineering, Brown University, Providence, RI 02912, USA \\ ${ }^{\mathrm{C} E}$ E.T.S. Enginyers de Camins, Canals i Ports, Technical University of Catalonia, \\ Campus Nord UPC, Mòdul C1, Gran Capitán s/n, 08034 Barcelona, Spain
}

\begin{abstract}
We present a finite element method with a finite thickness embedded weak discontinuity to analyze ductile fracture problems. The formulation is restricted to small geometry changes. The material response is characterized by a constitutive relation for a progressively cavitating elastic-plastic solid. As voids nucleate, grow and coalesce, the stiffness of the material degrades. An embedded weak discontinuity is introduced when the condition for loss of ellipticity is met. The resulting localized deformation band is given a specified thickness which introduces a length scale thus providing a regularization of the post-localization response. Also since the constitutive relation for a progressively cavitation solid is used inside the band in the post-localization regime, the traction-opening relation across the band depends on the stress triaxiality. The methodology is illustrated through several example problems including mode I crack growth and localization and failure in notched bars. Various finite element meshes and values of the thickness of the localization band are used in the calculations to illustrate the convergence with mesh refinement and the dependence on the value chosen for the localization band thickness.
\end{abstract}

Keywords: A. Ductile fracture modeling; B. Finite element methods; C. Embedded weak discontinuities; Localization of deformation; Regularization 


\section{Introduction}

In a cohesive approach to fracture, two independent constitutive relations are used; one characterizing the deformation behavior of the bulk material and another, for the fracture process, giving the response of one or more cohesive surfaces (or cohesive zones) ([Dugdale, 1960], [Barenblatt, 1962], [Hillerborg et al., 1976], [Needleman, 1987] and [Xu and Needleman, 1994]). The volumetric response is specified in terms of a stress-strain relation while the cohesive response is specified in terms of a traction-displacement relation that initially increases, attains a maximum and then decreases to zero thereby leading to the creation of new free surface. From dimensional considerations a characteristic length is introduced. A disadvantage of this approach is that the cohesive traction-separation relation does not directly involve the hydrostatic stress which plays a key role in the progressive cavitation process of ductile fracture. In order to overcome this limitation, Siegmund and Brocks (2000) have considered a cohesive strength that is hydrostatic stress dependent.

In another approach, a single volumetric constitutive relation is used which allows
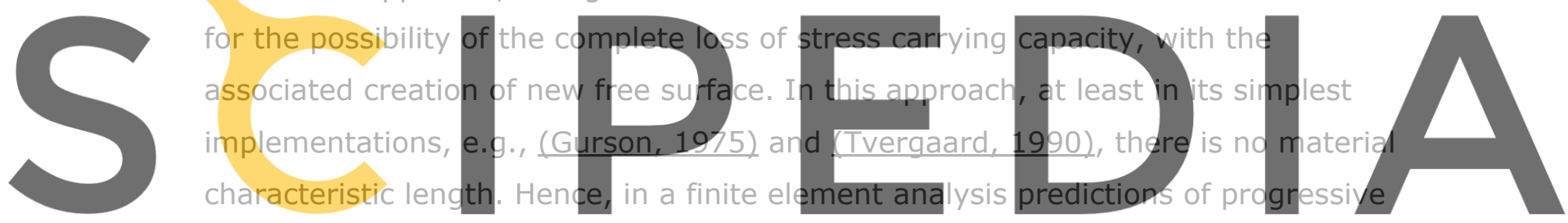

fracture are inherently mesh dependent. Nonlocal constitutive relations have been

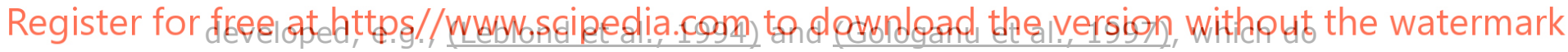

contain a characteristic length. Such a formulation is aimed at capturing size

dependence at the micromechanical scale. Accurate computations using such a constitutive relation require mesh sizes smaller than the characteristic length which is typically of the order of tens or a few hundred microns. In a component or structural level analysis of ductile fracture the computational cost for using such a fine mesh is generally prohibitive.

The formulation we present here is aimed at component or structural level analysis of room temperature ductile fracture of structural metals occurring by the nucleation, growth and coalescence of micro-voids. A characteristic feature of this model is that it is strongly influenced by the stress triaxiality. The size dependence that we aim to capture is that associated with the localization of deformation which, for a rate independent solid under quasi-static loading conditions, results in a loss of ellipticity of the governing equations so that the initial/boundary value problem becomes ill-posed. 
We use a standard finite element formulation and within each element monitor the sign of the determinant of the relevant acoustic tensor. When that determinant vanishes, the shape functions in the element are enhanced by additional functions which allow for a weak discontinuity band of finite thickness. Plastic deformation continues inside the band while elastic unloading takes place outside the band. The thickness of the localization band is considered to be a material property and is a prescribed quantity. Hence in our formulation the material characteristic length is presumed to be smaller than the element size. There is a large literature on embedding discontinuity modes, both weak and strong, in various contexts into finite element formulations, e.g., (Ortiz et al., 1987), (Moes et al., 1999), (Wells and Sluys, 2001), (Oliver et al., 2002), (Remmers et al., 2003) and (Belytschko et al. 2008). The finite element formulation here differs in having a finite thickness band. The post-localization response in the band is governed by the pre-localization constitutive relation which allows for the complete loss of stress carrying capacity and the creation of new free surface. Hence, in our formulation the discontinuity can transition from a weak discontinuity to a strong discontinuity, using a unified constitutive framework. Once the band forms, the net effect is similar to having a

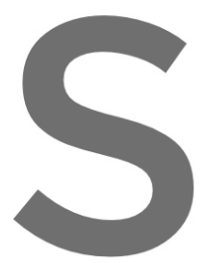
cohesive surface with a hydrostatic stress dependent cohesive lawthat energes
directly from the material's constitutive description. Although we focus attention on
ductile fracture aralyses and in particular on the Gurson (1975) framework, the
methodology can be directly used with any rate independent constitutive relation

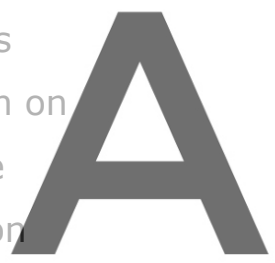
that permits a complete loss of stress carrying capacity.

Register for free at https//www.scipedia.com to download the version without the watermark

\section{Governing equations}

\subsection{Field equations}

Attention is restricted to small deformations so that geometry change effects are neglected. With body forces neglected, the principle of virtual work is written as

$$
\int_{\Omega} \boldsymbol{\sigma}: \nabla \delta \boldsymbol{u} d \Omega=\int_{\Gamma} \boldsymbol{t} \cdot \delta \boldsymbol{u} d \Gamma
$$

where $\delta$ denotes an admissible variation, $\Omega$ is a region bounded by $\Gamma, \boldsymbol{\sigma}$ is the stress tensor, $\boldsymbol{u}$ is the displacement vector, $\nabla$ is the gradient operator and

$$
\boldsymbol{t}=\boldsymbol{\sigma} \cdot \boldsymbol{v}
$$

with $\boldsymbol{v}$ normal to $\Gamma$.

The boundary conditions on $\Gamma$ are to prescribe either the traction $\boldsymbol{t}$ or the displacement $\boldsymbol{u}$. 
The strain tensor eis defined by

(3)

$\epsilon=\frac{1}{2}\left[\nabla \boldsymbol{\nabla}+(\nabla \boldsymbol{H})^{\bar{r}}\right]$

Here ()$^{T}$ denotes the transpose.

\subsection{Constitutive relation}

The constitutive relation used for the specimen is a modified Gurson ([Gurson, 1975], [Tvergaard, 1981], [Tvergaard, 1982] and [Tvergaard and Needleman, 1984]) constitutive relation for a progressively cavitating plastic solid that has been extensively used to model ductile damage and is briefly outlined here. Attention is confined to small deformations, i.e., geometry changes are neglected, and to rate independent matrix material behavior. Background on the (Gurson (1975)) framework and further details are given in (Tvergaard, 1990) and (Needleman et al., 1992). Some recent ductile fracture modeling studies are presented in (Lorentz et al., 2008), (Li and Karr, 2009) and (Bai and Wierzbicki, 2008).

The constitutive relation used is a small deformation specialization of a rate

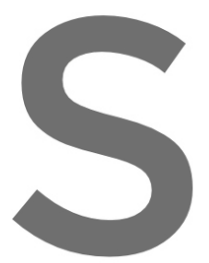
independent modified Gurson constitutiv
written as the sunn of an elastic strain rat
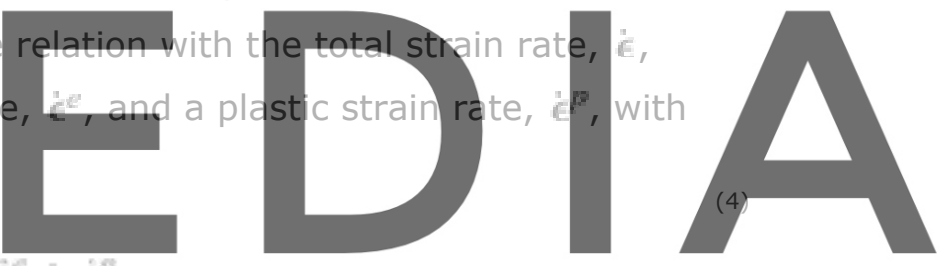

Register for ffireeceat https//www.scipedia.com to download the version without the watermark

$$
\dot{\epsilon}^{e}=C^{e-1}: \dot{a}
$$

with

$$
C^{e}=\frac{E}{1+y} I+\frac{1 E}{(1+1)(1-2 y)} \mathbf{I} \otimes 1
$$

Here, (') denotes the rate of change with respect to some monotonically increasing time-like parameter, $\boldsymbol{C}^{e}$ is the tensor of isotropic elastic moduli with Young's modulus $E$ and Poisson's ratio $v, \boldsymbol{I}$ and lare, respectively, the fourth order and second order identity tensors.

The plastic strain rate, $\dot{\boldsymbol{\epsilon}}^{p}$, is given by

$$
\dot{e}^{y}=\dot{A} \frac{\partial \dot{\beta}}{\partial a}=\dot{A} P
$$


in terms of the yield criterion

$$
\phi=\frac{\sigma_{e}^{2}}{\sigma^{2}}+2 q_{1} f^{t} \cosh \left(\frac{3 q_{2} \sigma_{R^{4}}}{2 \sigma}\right)-\left(q_{1} f^{\mathrm{t}}\right)^{2}-1=0
$$

where $f^{*}$ is a specified function of the void volume fraction $f$ and

$$
\sigma_{s}=\sqrt{\frac{2}{2} S: S,} \quad S=G-\sigma_{n t} 1, \quad \sigma_{n s}=\frac{1}{3} \operatorname{tr}(a)
$$

Here, $\operatorname{tr}$ denotes the trace.

The parameters $q_{1}$ and $q_{2}$ were introduced by (Tvergaard, 1981) and (Tvergaard, 1982 ) and the function $f^{*}$, which accounts for the effects of rapid void coalescence at failure through the model parameters $f_{c}$ and $f_{f}$ (Tvergaard and Needleman, 1984), is given by
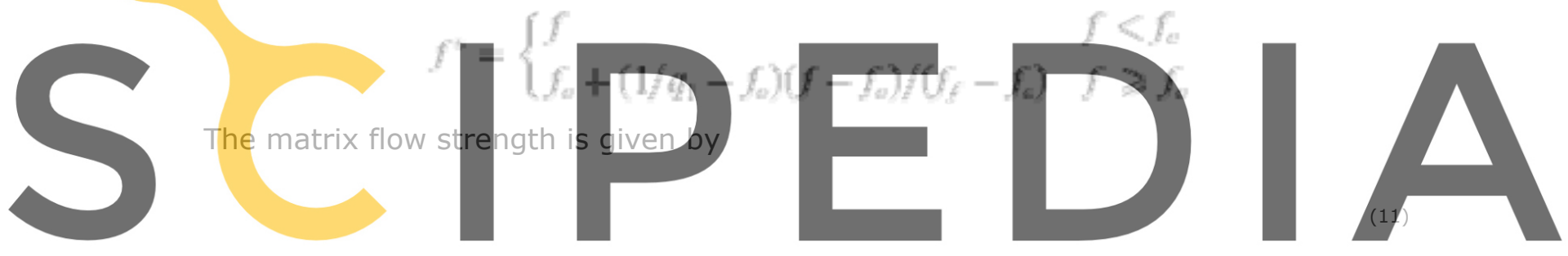

Register for free at https//www.scipedia.Compto download the version without the watermark

where $\sigma_{y}$ is the initial yield strength, $N$ is the strain hardening exponent and $\bar{C}$ is the matrix equivalent plastic strain and its rate is related with the macroscopic plastic work rate through

$$
(1-f) \sigma \epsilon=\sigma: e^{p}
$$

The initial void volume fraction is taken to be $f_{0}$ and the evolution of the void volume fraction is governed by

$$
\vec{f}=(1-f) \operatorname{tr}\left(\hat{e}^{f}\right)+\vec{f}_{\text {nex }}
$$

The first term on the right-hand side of Eq. (13) accounts for void growth and the second term for void nucleation.

Consideration here is restricted to plastic strain controlled nucleation with (Chu and Needleman, 1980) 


$$
\hat{f}_{\text {Red }}=\hat{f}_{2} \quad f=\frac{f_{N}}{s_{N} \sqrt{2 \pi}} \exp \left[-\frac{1}{2}\left(\frac{\varepsilon-\epsilon_{N}}{s_{N}}\right)^{2}\right]
$$

where $f_{N}, s_{N}$ and $e_{N}$ are material parameters.

For plastic loading the plastic strain rate $\dot{e}^{p}$ can be written as ([Gurson, 1975] and [Needleman and Rice, 1978])

$$
\dot{\varepsilon}=\frac{\dot{E}: \dot{\sigma}}{E} p
$$

where
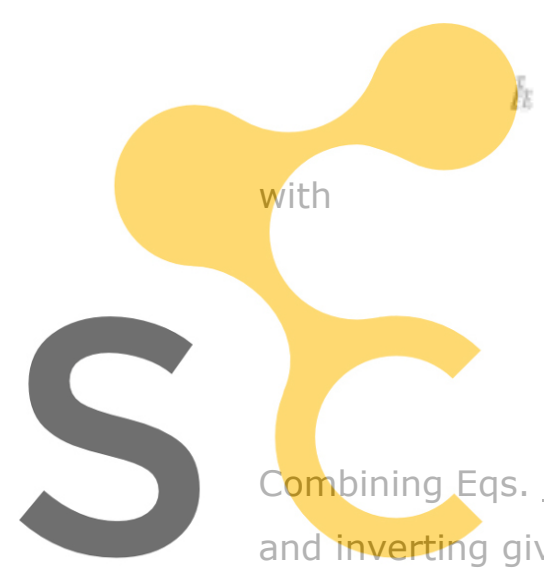

$$
f=-\left[(1-f) \frac{\partial \phi}{\partial f} \operatorname{tr}(\rho)+\left(\frac{\partial \phi}{\partial f} \partial+\frac{\partial \phi}{\partial \sigma}\right) \frac{\sigma: \underline{p}}{(1-f) \sigma}\right]
$$

with
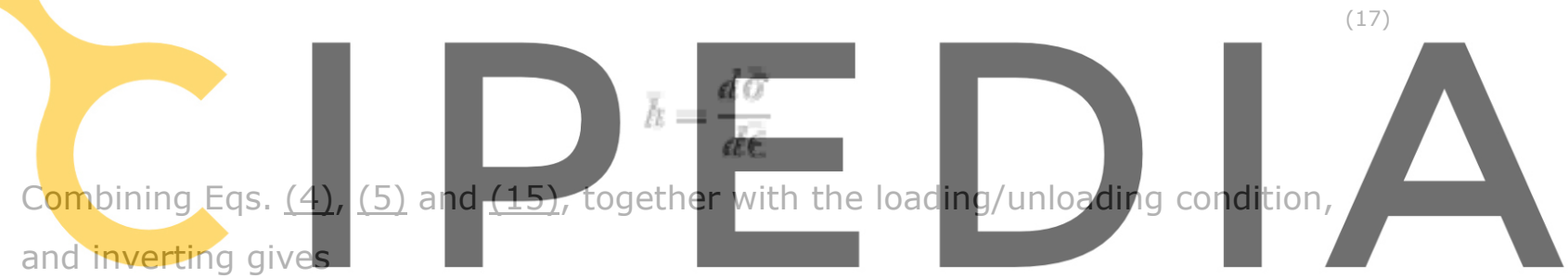

Register for free at https//www.scipedia.com to download the version without the watermark

$$
\dot{a}=\mathrm{C}: \dot{c}
$$

$$
C=\left\{\begin{array}{llll}
C^{*} & \text { if } \phi<0 \text { or } \phi=0 & \text { and } \frac{p: \dot{\sigma}}{E}<0 & \text { (elastic unloating) } \\
C^{*} & \text { if } \phi=0 \text { and } \frac{p: \dot{\sigma}}{E}>0 & \text { (plastiv loading) }
\end{array}\right.
$$

where $\boldsymbol{C}^{e}$ is given by Eq. (6), $\boldsymbol{C}^{p}$ is given by

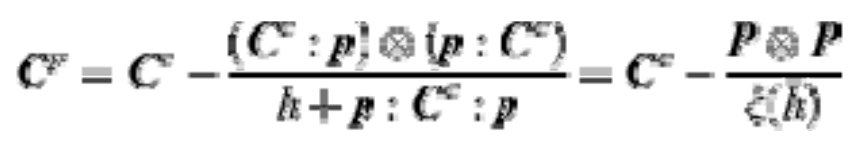

and $\boldsymbol{P}=\boldsymbol{C}^{e}: \boldsymbol{p}=\boldsymbol{p}: \boldsymbol{C}^{e}$. Both $\boldsymbol{C}^{e}$ and $\boldsymbol{C}^{p}$ have major symmetries. 


\section{Localization analysis}

The localization analysis is based on the work of (Hadamard, 1903), (Thomas, 1961), (Hill, 1962) and (Rice, 1976). Explicit results for the Gurson (1975) and modified Gurson solids are given in (Needleman and Rice, 1978), (Sánchez et al., 2008) and (Saje et al., 1982).

We consider a band across which there is a possible jump in the displacement rate gradient but the displacement rates remain continuous. The structure of this jump must be a tensor of rank one, given by

\section{$[\nabla$ 내 $=30$ H}

where denotes a jump, $\boldsymbol{n}$ is the band normal and $b$ an arbitrary velocity vector.

Incremental equilibrium across the band requires
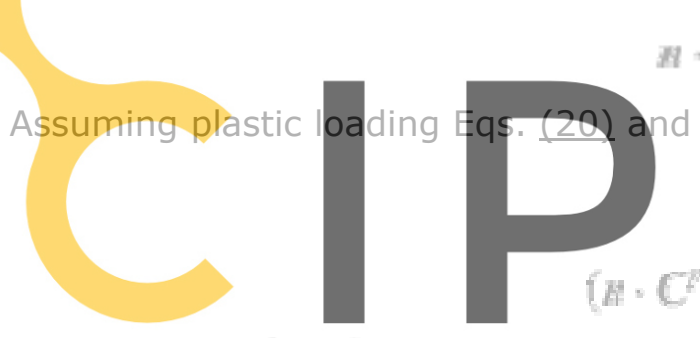

$n \cdot \mid \vec{a}]=\theta$

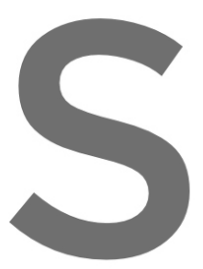

Hence, either $\mathrm{g}=0$ or

Register for free at https//www.scipedia.com to download the version without the watermark

$$
\operatorname{det}\left(\boldsymbol{n} \cdot \boldsymbol{C}^{p} \cdot \boldsymbol{n}\right)=0
$$

As shown in (Oliver et al., 2004) and (Sánchez et al., 2008), Eq. (23) can be written as

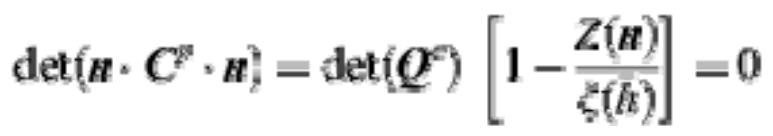

where $\xi$ is defined in Eq. (19), $\boldsymbol{Q}^{e}=\boldsymbol{n} \cdot \boldsymbol{C}^{e} \cdot \boldsymbol{n}$ and

$$
Z(n)=\boldsymbol{H} \cdot \boldsymbol{P} \cdot Q^{2-1} \cdot \boldsymbol{P} \cdot \boldsymbol{H}=\frac{2(1+y)}{E}(\boldsymbol{H} \cdot \boldsymbol{P} \cdot \boldsymbol{P} \cdot \boldsymbol{H})-\frac{1+y}{E(1-y)}[\boldsymbol{i} \cdot \boldsymbol{P} \cdot \boldsymbol{H})^{2}
$$


Since

$$
\operatorname{del}\left(Q^{e}\right)=\frac{E\left(1-v^{2}\right)}{\left(1+v^{2}\right)(1-2 v)}\left[\frac{E}{2\left(1+v^{2}\right)}\right]^{2}
$$

is positive for $E>0$ and $-1 \leqslant v \leqslant 1 / 2$, a weak discontinuity is possible when

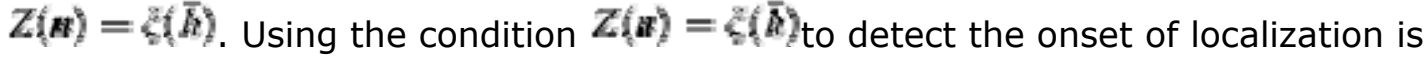
computationally convenient.

After localization occurs, continued plastic loading takes place in the band which is presumed to have a uniform strain and stress state, and elastic unloading occurs outside the band.

A connection can be made with a cohesive surface approach. Denote the total

volume under consideration by $\Omega$, a band volume $\Omega_{D}$ of thickness $D$ and bounded by two surfaces $\Gamma_{D}^{+}$and $\Gamma_{D}^{-}$across which a jump in the displacement rate gradient

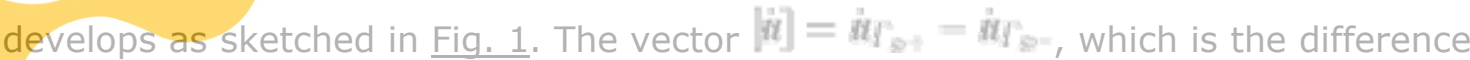
between the velocity vector on the boundary $\Gamma_{D}{ }^{+}$and that on $\Gamma_{D}^{-}$is introduced.

Denoting the volume outside $\Omega_{D}$ by $\Omega_{0}$, the stress work rate is given by
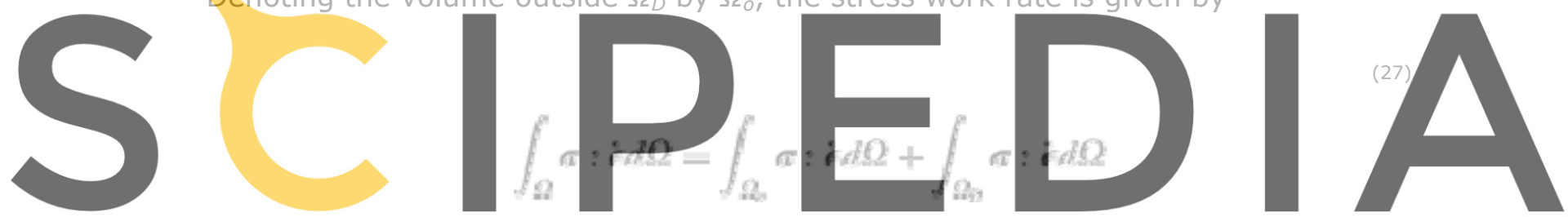

From the equilibrium condition ( $\nabla \cdot \sigma=0$ ) in $\Omega_{0}$ and $\Omega_{D}$, traction continuity Eq. (21)

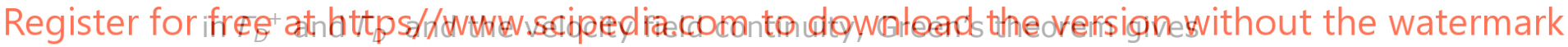

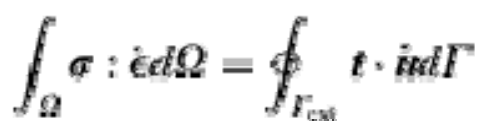

where $\boldsymbol{t}=\boldsymbol{\sigma} \cdot \boldsymbol{v}$ denotes the traction vector on the external surface $\Gamma_{\text {ext }}$ of $\Omega$.

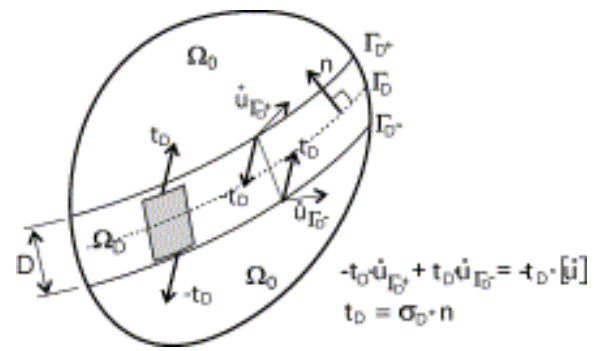

Fig. 1. Connection of the finite thickness band method with the cohesive surface approach. 
Focussing on discontinuities of displacement gradient rate that are localized in domains $\Omega_{D}$ having a small thickness $D(D \rightarrow 0)$ and since the band is taken to be homogeneously deformed, the stresses are uniform across $\Omega_{D}$ so that, from Green's theorem, the second term of Eq. (27) can be written as

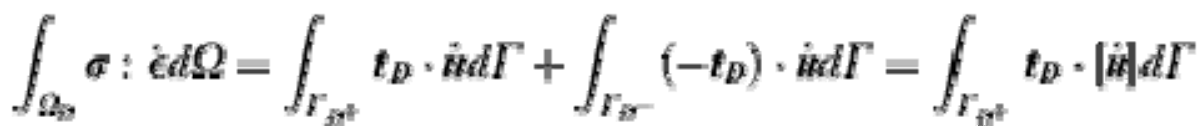

where any contribution along the edge of the band is neglected.

The relations in Eqs. (27), (28) and (29) can be rewritten as

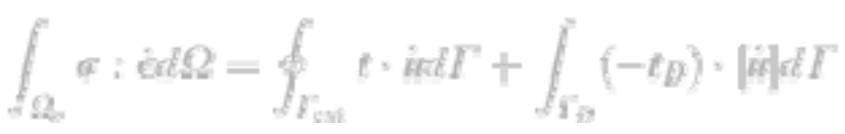

where the last term can be interpreted as the work rate produced by cohesive tractions $\left(-t_{D}\right)$ in $\Omega_{0}$, acting on a $\Omega_{D}$-representative mean surface $\Gamma_{D}$, in between $\Gamma_{D}{ }^{+}$ and $\Gamma_{D}^{-}$, which are induced by stresses in $\Omega_{D}$. Thus, by using a porous plastic

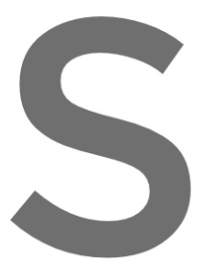
constitutive relation, the effect of stress triaxiality in a localized band is accounted
for. The band thickness $D$ now enters the formulation as a material parameter to be
specified. By way of contrast, in a cohesive surface formulation the characteristic
length enters through a constitutive relation for $t$ on $\Gamma_{D}$. Furthernore, since the
stress carrying capacity in the band vanishes when $f=f_{f}$ creating new free surface,

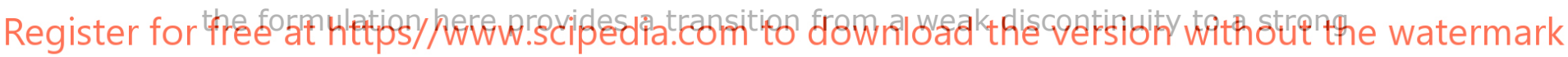
discontinuity.

\section{Finite element implementation}

The computational procedure is based on a finite element formulation with embedded weak discontinuity modes. The key features of the formulation are the finite thickness of the band and the use of a tracking algorithm to obtain geometrical continuity of the band across the finite element mesh. The implementation is confined to two dimensions and is a direct extension of the nonsymmetrical, kinematical and statically optimal four node quadrilateral finite element formulation with embedded strong discontinuities described in Oliver et al. (2003). Additional aspects of a similar finite element technology can be found in (Manzoli and Shing, 2006) and (Linder and Armero, 2007). A sketch of the implementation, in the context of a finite thickness band method, is briefly described here. 
Let a body $\Omega$ having a strain discontinuity finite band of thickness $D$ and orthogonal to $\boldsymbol{n}$, as shown in Fig. 2(c), be given. We use $\Omega^{+}$and $\Omega^{-}$to denote the two parts of the body divided by this finite thickness band.

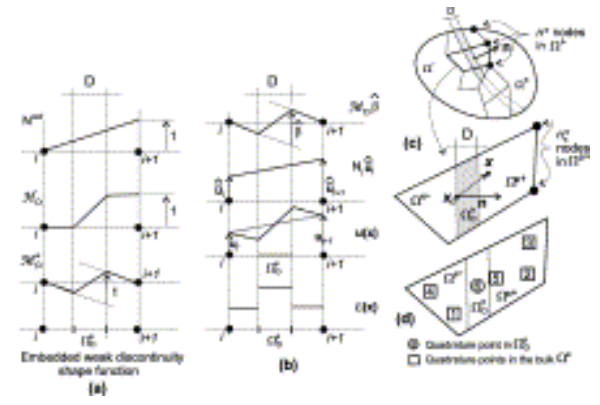

Fig. 2. Weak discontinuity kinematics used in the finite element formulation. (a) Shape

functions of the embedded weak discontinuity mode $k_{k}^{e} x$. (b) Displacement and strain fields. (c) Finite element with a weak discontinuity band displaying the nodes $v_{e}^{+},(d)$ The quadrature points for the integration rule.
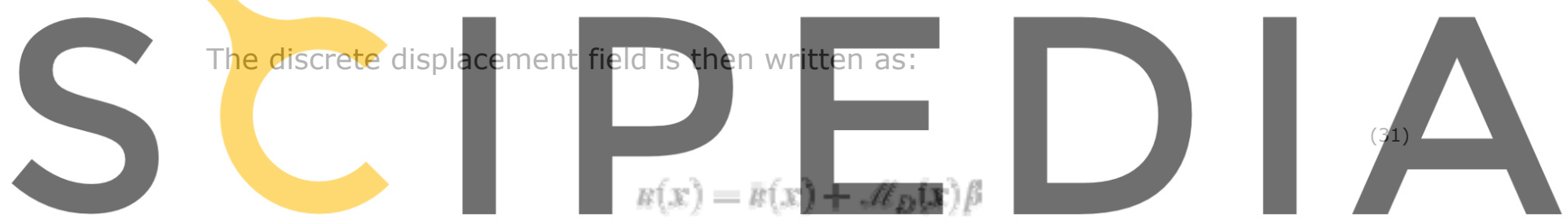

where $\overrightarrow{a f} x$ represents a smooth part of the displacement field that, in the domain

Register for free at https//www.scipedia.com to download the version without the watermark

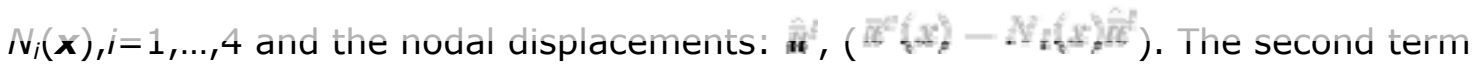
in Eq. (31) is the embedded weak discontinuity mode which is proportional to the displacement jump vector across the band: $\boldsymbol{\beta}$. In finite element $e$, this term is interpolated by:

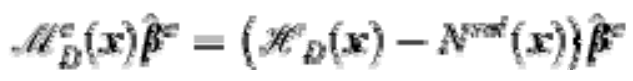

where $N^{\text {sol }}$ comes from the addition of the shape functions $N_{i}$ corresponding to the

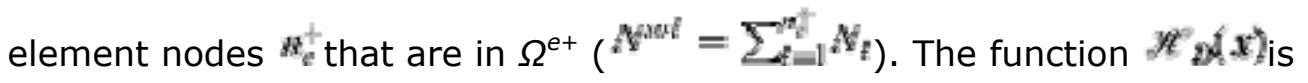

$$
\mathscr{H}_{D}(x)= \begin{cases}0 & \forall x \in \Omega^{e-} \\ {\left[\frac{1}{b}\left(\left(x-x_{0}\right) \cdot n\right)\right]} & \forall x \in \Omega_{D}^{e} \\ 1 & \forall x \in \Omega^{e+}\end{cases}
$$


with $x_{o}$ an arbitrary point on the border $f Q^{-} \cap \Omega_{b}^{e}$ and $D$ the specified band thickness.

The one-parameter interpolation in Eq. (32) provides a weak discontinuity mode with a constant displacement jump per element. Fig. 2(a) displays the shape function $\mathbb{H}_{0}^{e} x_{\text {for }}$ the one-dimensional case, and Fig. 2(b) shows the corresponding displacement and strain fields determined by Eq. (31).

The strain $\epsilon$, compatible with the displacement Eq. (31), is evaluated by defining a generalized strain-displacement matrix $\boldsymbol{B}$ and displacement vector $\hat{\boldsymbol{u}}$, such that the discrete strain-displacement relation reads

$$
\begin{aligned}
& \epsilon=B \text { ik }
\end{aligned}
$$

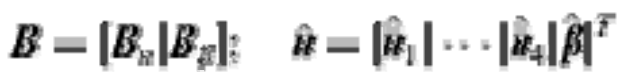

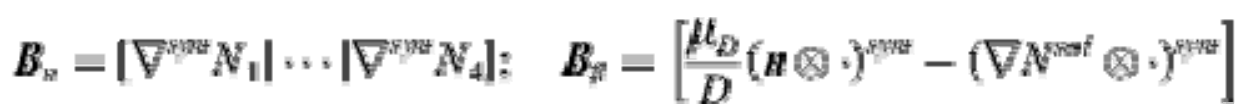

The coefficient $\mu_{D}$ is 0 or 1 depending if the strain evaluation point is outside or inside $\Omega_{b}^{e}$, respectively, and $\nabla^{s y m}$ is the symmetric gradient operator. The standard B-bar technique which involves modifying the matrix $\boldsymbol{B}$, see Hughes (1980), is used in order to prevent locking caused by possible isochoric deformations. The modifications proposed by the B-bar technique apply to those terms related with $\nabla$ ${ }^{s y m} N_{i}$ and $\nabla N^{\text {sol }}$ in $\boldsymbol{B}_{u}$ and $\boldsymbol{B}_{\beta}$ while the term multiplied by $\mu_{D}$ remains unchanged. Following the Petrov-Galerkin approach of Oliver et al. (2003), the equilibrium equations are written as follows

$$
\int_{\Omega} B_{r}^{T} \sigma d \Omega=F^{e s t}
$$

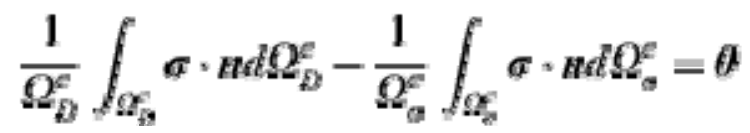

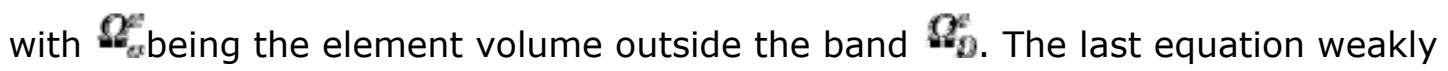
imposes the equilibrium condition Eq. (21) across the band.

The numerical integration of the terms in Eqs. (35) and (36) is performed by a six point integration rule, as depicted in Fig. 2(d). The role specified for each quadrature point, is summarized as follows: 
(i) The standard four point Gaussian quadrature rule, for the bilinear quadrilateral elements, specifies the position and weights of the Gauss Points $(1, \ldots, 4)$. The terms in Eq. (35) are evaluated using these four points, specifying $\mu_{D}=0$ in Eq. (34).

(ii) Gauss points five and six are at the same geometrical position, the center of the element.

(iii) The two terms in Eq. (36) are integrated using a one-point integration rule: Gauss point 5 is used for the second term and Gauss point 6 for the first term. The stress $\boldsymbol{\sigma}_{D}$, using the strain $E_{D}$, by setting $\mu_{D}=1$ in Eq. (34) and the constitutive relation of Section 2.2, is computed at Gauss point 6 . While the stress $\boldsymbol{\sigma}_{\Omega 0}$, with $\mu_{D}=0$ is computed at Gauss point 5 . The traction $\boldsymbol{t}_{D}$ in $\Gamma_{D}$, Eq. (30), does not appear explicitly in the formulation. Even though, it could be obtained from the projection $\left(\boldsymbol{\sigma}_{D} \cdot \boldsymbol{n}\right)$.

(iv) Gauss points 5 and 6 have the same stress-strain states prior to the activation of the weak discontinuity mode.

Additional numerical implementation aspects and features are:

(1) The localization condition $Z(m)-s(\bar{h})$ is monitored in each finite element (at Gauss point 5 which is at the geometrical finite element center) using the procedure given by Oliver and Huespe (2004). The orientation $\boldsymbol{n}^{\circ}$ is defined by

$$
\boldsymbol{n}^{\circ}=\cos \left(\theta^{\circ}\right) \boldsymbol{P}_{I}+\sin \left(\theta^{\circ}\right) \boldsymbol{P}_{I I I}
$$

$$
\begin{gathered}
\tan ^{2}\left(\theta^{\circ}\right)=-\frac{P_{H F f}-\sigma o P_{F}}{P_{F}-\sigma o P_{H F}} ; \quad\left(\theta^{\circ}=0 \text { if } P_{G H}>\phi \partial P_{i}\right) \\
\omega=\frac{v}{\left(1-v^{2}\right)}
\end{gathered}
$$

where $\left\{P_{I}>P_{I I}>P_{I I I}\right\}$ and $\left\{\boldsymbol{P}_{I}, \boldsymbol{P}_{I I}, \boldsymbol{P}_{I I I}\right\}$ are the eigenvalues and eigenvectors of $\boldsymbol{P}$, respectively, with $\boldsymbol{P}=\boldsymbol{C}^{e}: \boldsymbol{p}$ and $\boldsymbol{p}$ defined in Eq. (7). Thus, the bifurcation detection procedure consists of evaluating Eqs. (37) and (38) and $Z_{o}=Z\left(\boldsymbol{n}^{\circ}\right)$ at any stage of a plastic loading process and comparing $Z_{o}$ with $\overline{s(\bar{B})}$. A localization bifurcation occurs when $\left.Z_{w}-\delta \bar{f} \bar{h}\right\rangle_{\text {and }}$, therefore, $\boldsymbol{n}^{\text {crit }}=\boldsymbol{n}^{\circ}$.

(2) When the localization condition is first satisfied for some band normal $\boldsymbol{n}$ at one of these Gauss points, the bulk Gauss points $(1, \ldots, 5)$ are required to undergo elastic unloading, while plastic loading continues at Gauss point 6 . Thus, the weak discontinuity mode, Eq. (32), becomes active allowing the development of plastic loading in the band that is compatible with elastic unloading outside the band. (3) In the present numerical implementation, a fundamental ingredient is the geometric continuity of the weak discontinuity mode across the finite element mesh. In general, Eq. (38) gives rise to two distinct localization angles. At the initiation of a localization band, by means of numerical experimentation, one of these angles is chosen as the one leading to the less dissipative response.

Subsequently the detection of the nodes $k_{e}^{+}$is determined through a discontinuity tracking algorithm following the global approach presented in Oliver et al. (2004). (4) The degrees of freedom $\boldsymbol{\beta}^{e}$ corresponding to the weak discontinuity modes are local to each element and can therefore be condensed at the element level to increase the computational efficiency.

The methodology was implemented in the COMET finite element code, see Cervera

et al. (2001). 


\section{Numerical examples}

\subsection{Localization band}

The rectangular bar sketched in the insert of Fig. $3(a)$, is subject to prescribed displacement loading in the $x_{2}$-direction corresponding to plane strain tension. The sides $x_{1}=-b / 2, b / 2$ are traction free. The material parameters used in the calculation are: $\sigma_{y} f E=0.0025_{0} g=0.3, N=0.1, \sigma_{1}=1.5, f_{2}=1.0, f_{\varepsilon}=0.15$ and $f_{f}=0.25$. The initial void volume fraction is $f_{0}=0.005$ and void nucleation is characterized by $\epsilon$ ${ }_{N}=0.3, f_{N}=0.04$ and $s_{N}=0.1$. The specimen dimensions are $2 b$ parallel to the loading axis and $b$ perpendicular to the loading axis. Since geometry changes are neglected, the localization results presented here are not aimed at modeling material behavior but are aimed at illustrating the capability of the numerical method. A bifurcation analysis gives that localization initiates at $\epsilon_{\text {crit }}=0.205$ and that the localization band forms at an angle of $40^{\circ}$. Prior to the onset of localization, deformation is uniform with a stress triaxiality ratio, $T=\sigma_{m} / \sigma_{e}$, of $T=0.57$.

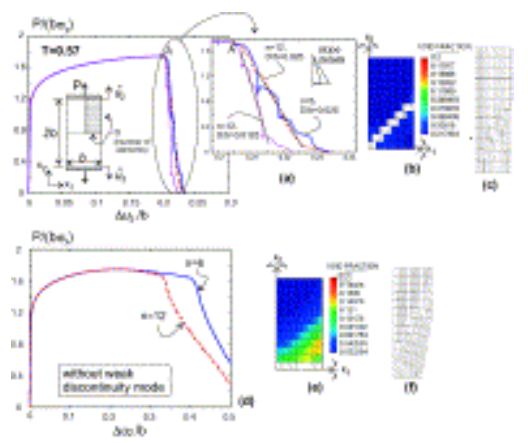

Fig. 3. Localization and failure in plane strain tension. (a) Normalized load versus axial strain obtained using the weak discontinuity formulation for $D / b=0.0125$ and $D / b=0.025$ with $n=6,12$. The inserts show a sketch of the specimen analyzed and details of the post-localization response. (b) Contours of void volume fraction $f$ showing the localization and failure mode at $\Delta u_{2} / b=0.215$; (c) Deformed mesh at $\Delta u_{2} / b=0.215$. (d-f) Results obtained using a formulation without including weak discontinuity modes with (d) normalized load versus axial strain. (e) Contours of void volume fraction $f$ showing the localization and failure mode at $\Delta u_{2} / b=0.457$. (f) Deformed mesh at $\Delta u_{2} / b=0.457$. The void fraction contours in white show finite elements for which $f>0.2$.

Curves of load versus applied displacement are shown in Fig. 3(a) for two values of the thickness $D, D / b=0.0125$ and $D / b=0.025$. Quadrilateral bilinear displacement 
elements are used with an $n \times n$ element mesh so that each element has an aspect ratio of two which is an unfavorable aspect ratio for resolving localization in a $40^{\circ}$ band. The onset of localization takes place at a smaller strain than $\varepsilon_{N}$ and is well represented in all calculations in Fig. $3(a)$. The post-localization response is relatively independent of the mesh resolution, it depends only on the value of $D$. In Fig. 3 (b), contours of void volume fraction are plotted which show the orientation of the localization band for the $n=12$ calculation with $D / b=0.0125$, and Fig. 3(c) displays the deformed mesh at $\Delta u_{2} / b=0.215$.

Without including the enhanced modes in the finite element formulation, localization initiates at $\epsilon_{\text {crit }}=0.328$ (with $n=12$ ), see Fig. $3(d)$, and the band angle is perpendicular to the loading direction, Fig. 3(e). The deformed mesh at $\Delta u_{2} / b=0.457$ is shown in Fig. 3(f). As seen in Fig. 3(d), the post-localization response depends strongly on the mesh resolution.

\subsection{Mode I crack growth}

We consider small scale yielding of a mode I crack as displayed in Fig. 4 . The exterior boundary of the domain analyzed is subject to imposed displacements that are compatible with the mode I linear elastic singular crack tip field which are given by

$$
t_{1}=\frac{2(1+1) K_{F}}{E} \sqrt{\frac{F}{2 \pi}} \cos (\theta / 2)\left[1-2 v+\sin ^{2}(\theta / 2)\right]
$$

$$
\omega_{2}=\frac{2\left(1+v^{2}\right) K_{I}}{E} \sqrt{\frac{F}{2 \pi}} \sin (\theta / 2)\left[2-2 v-\cos ^{2}(\theta / 2)\right]
$$

where $r=\sqrt{x_{1}^{2}+x_{2}^{2}}$ and $\theta=\tan ^{-1} x_{2} / x_{1}$ and traction free conditions are imposed along the crack faces. Under small scale yielding conditions, the stress intensity factor $K_{I}$ is related to the Rice's (Rice, 1968) J-integral $I=K_{\tilde{*}}^{2}\left(1-\mathrm{V}^{2}\right) / E$.

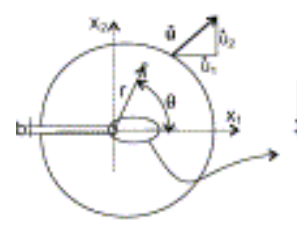

(a)

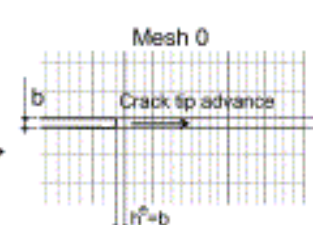

(b) 
Fig. 4. (a) Sketch of the small scale yielding crack growth problem analyzed. (b) Mesh 0 is used in an analysis without any weak discontinuity modes; $b$ is the side length of the square elements along the initial crack plane.

The material parameters used in the analysis are: $\sigma_{y} / E=0.0025, v=0.3, N=0.1$, $q_{1}=1.5, q_{2}=1.0$. The initial void volume fraction is $f_{0}=0.005$ and void nucleation is characterized by $\epsilon_{N}=0.3, f_{N}=0.04$ and $s_{N}=0.1$. In the calculations in this Section we take $f^{*} \equiv f$.

Two types of calculations are carried out: (i) three meshes are used to compute crack growth resistance curves, curves of $J$ versus the amount of crack growth $\Delta a$, for three values of the characteristic length parameter $D$ as displayed in the inserts in Figs. 5(a)-(c); and (ii) for comparison purposes a calculation without any weak discontinuity modes using the mesh termed Mesh 0 in Fig. 4. In Mesh $0, b$ is the side length of the square elements along the initial crack plane and hence also the initial crack tip opening. The calculation with Mesh 0 can be regarded as a "cell" calculation, Xia and Shih (1995), with cell size $b$.

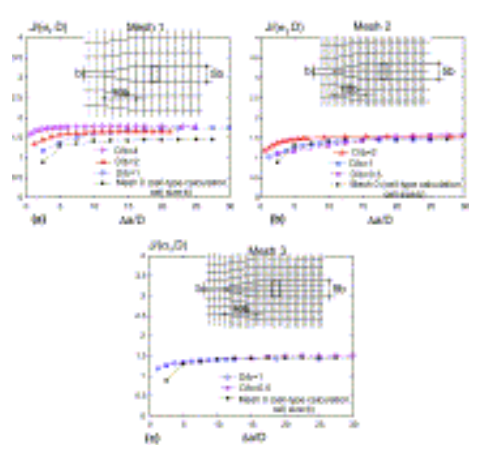

Fig. 5. Normalized curves of $J$ versus the amount of crack growth $\Delta a$ for various values of the characteristic length $D$ and for various finite element meshes. (a) Mesh 1; element size $2.5 b \times 5 b$. (b) Mesh 2; element size $1.25 b \times 5 b / 2$. (c) Mesh 3; element size $1.25 b \times 5 b / 3$. For comparison purposes a calculation without weak discontinuity modes is shown (Mesh 0 ). In this case, the normalization $D=b$ is used.

The initial crack tip opening is also $b$ for the three meshes in Fig. 5 but the mesh height is rapidly increased to $5 b$ in Mesh 1 , to $5 b / 2$ in Mesh 2 and to $5 b / 3$ in Mesh 3. For all three meshes, the orientation of the localization band is consistent with crack growth along the initial crack plane. Without any additional localization modes, the plastic dissipation associated with fracture and hence the crack growth 
resistance is sensitive to the height of the elements along the initial crack plane. The width of the finite elements along the crack plane also is of significance in correctly capturing the steep stress gradients ahead of the crack tip.

Normalized crack growth resistance curves, $J /\left(\sigma_{y} D\right)$ versus $\Delta a / D$, are plotted in

Fig. 5. In each of Figs. 5(a)-(c) results are shown for several values of $D$. For comparison purposes, the Mesh 0 calculation without result any weak discontinuity modes (the result for the Mesh 0 calculation is normalized by $D=b$ ) is shown in each plot. Due to the scaling of the axes, all curves in Fig. 5 should be the same for all values of $D$ and for all the finite element meshes. Except for an initial transient, this is essentially the case in Fig. 5(b) and (c). For the crudest mesh, Mesh 1 in Fig. $\underline{5}(a)$, there is a greater variation with the value of $D$ and also the "steady state" value of the crack growth resistance differs from that with the cell-type calculation using Mesh 0.

Fig. 6 shows curves of normalized $J$ versus the amount of crack growth $\Delta a$ with $D / b=1$ using Meshes 1, 2 and 3. For comparison purposes, the calculation without any weak discontinuity modes is shown (the Mesh 0 cell-type calculation which is normalized using $D=b$ ). The good agreement in Fig. 5 and Fig. 6 between the results for Mesh 0 , where there is a conventional implementation of the constitutive response, and those for Mesh 2 and Mesh 3 suggests that the loading-unloading behavior is correctly represented in the calculations using weak discontinuity modes. It is also worth noting that the crack growth calculations here represent a situation where there is a transition from a weak discontinuity to a strong discontinuity. Also, although similar results are obtained with the cell model Mesh 0 calculations, an advantage of our formulation is that the finite element discretization and the material characteristic length are specified independently.

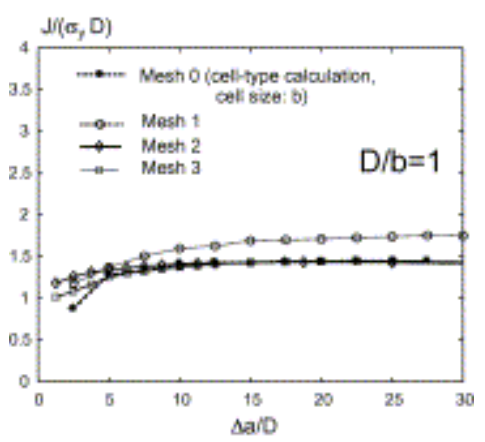

Fig. 6. Normalized curves of $J$ versus the amount of crack growth $\Delta a$ for Meshes 1 , 
2 and 3 with $D / b=1$. For comparison purposes a calculation without weak

discontinuity modes is shown (Mesh 0 ). In this case, the normalization $D=b$ is used.

\subsection{Ductile fracture in notched bars}

There are numerous works analyzing the ductile rupture of notched bars by means of numerical solutions, for example, (Needleman and Tvergaard, 1984), (Becker et al., 1988) and (Besson et al., 2001). In particular, (Besson et al., 2001) and (Besson et al., 2003) utilize the localization bifurcation criterion as a localization indicator as we do. Since geometry changes are neglected in the calculations here, our aim is not to analyze ductile fracture phenomena but to illustrate the capability of our methodology to capture curved cracks. We carry out calculations for the two notch geometries considered by Needleman and Tvergaard (1984) but restrict attention to plane strain conditions. Fig. 7 shows the geometries of the A-notch and D-notch bars along with the near notch finite element meshes used. The A-notch finite element mesh has 792 elements with their size in the strain localization zone being $h^{e} / b \approx 0.0250$. The D-notch finite element mesh has 1132 elements, with element sizes $h^{e} / b=0.020-0.028$ in the strain localization zone.

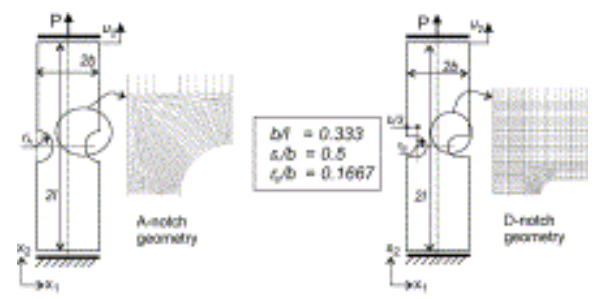

Fig. 7. Geometry of the A-notch and D-notch specimens and the finite element meshes used.

The material parameters are $\sigma_{y} / E=0.0033, v=0.3, N=0.06, q_{1}=1.5, q_{2}=1.0$ and void nucleation is characterized by $\epsilon_{N}=0.3, f_{N}=0.04$ and $s_{N}=0.1$. The initial void volume fraction is taken to be $f_{0}=0.0001$ and the void coalescence parameters in Eq. (10) are $f_{c}=0.15$ and $f_{f}=0.25$.

Fig. 8 displays curves of normalized average stress versus the average axial strain for the two notch geometries. For comparison purposes, corresponding results from Needleman and Tvergaard (1984) (where geometry changes were accounted for) are also plotted. We also show results obtained from calculations where no weak discontinuity modes were added. The effect produced by varying the characteristic 
band thickness $D$ on the overall response is shown in Fig. $8(\mathrm{~b})$ for the D-notch geometry specimen.

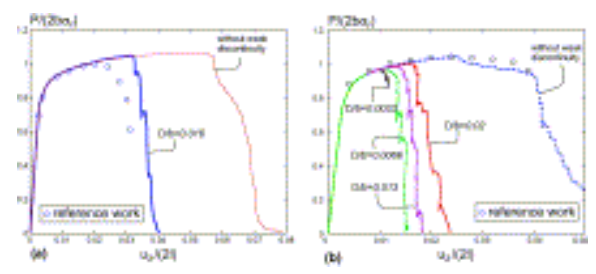

Fig. 8. Curves of load versus applied displacement for notched bars in plane strain. (a) A-notched bar. (b) D-notched bar.

Because the band angle rendering the acoustic tensor singular is not unique in general, a choice is involved in selecting the direction of the weak discontinuity mode. Hence, alternative modes of propagation are possible. The solution computed with the present numerical technique corresponds to a possible failure mode but from a single calculation it cannot be ascertained that the mode chosen is the one that gives the smallest failure strain and/or the minimum energy dissipation.

Contours of void volume fraction $f$ are shown in Fig. 9 and Fig. 10. In the early stages of deformation, the largest values of $f$ are at the notch root which is where the localization condition is first met. A shear band then propagates from this point at an angle of approximately $135^{\circ}$ with the $x_{1}$-axis. But it stops at a distance of approximately, $0.1 b$ for the D-notch and $0.16 b$ for the A-notch specimens, respectively. A new band develops at a direction of approximately $30^{\circ}-45^{\circ}$ with the $x_{1}$-axis intersecting the center of the bar. The D-notch bar shows a smoother transition from one localization band to the other. In this case, the band in the transition zone is not a shear band but a mixed mode band with a strong mode I component. Various stages of crack growth are displayed in Figs. 9 (a)-(d) and Figs. $\underline{10}(a)-(d)$, respectively, for both notch geometries. Also, in these figures the deformed meshes in the end of analysis are shown. 


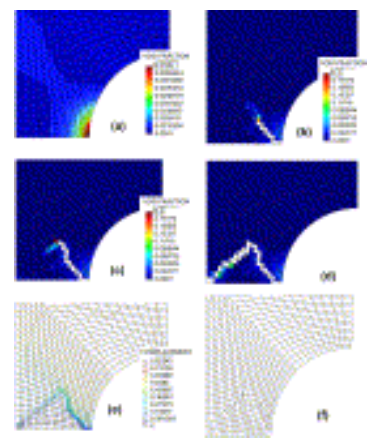

Fig. 9. Field quantity distributions and a deformed mesh showing the evolution of material failure for the A-notch geometry with $D / b=0.016$. (a) Void volume fraction distributions at $P / 2 b \sigma_{y}=0.98, \Delta u_{2} / I=0.020$. This coincides with the first time that material instability is detected in the notch root. (b) Void volume fraction distributions at $P / 2 b \sigma_{y}=0.72, \Delta u_{2} / I=0.036$. (c) Void volume fraction distributions at $P / 2 b \sigma_{y}=0.39, \Delta u_{2} / I=0.037$. (d) Void volume fraction distributions at $P / 2 b \sigma_{y}=0.016$, $\Delta u_{2} / I=0.040$. (e) Distribution of $u_{2}$ at $P / 2 b \sigma_{y}=0.016, \Delta u_{2} / I=0.040$. (f) Deformed mesh at $P / 2 b \sigma_{y}=0.016, \Delta u_{2} / I=0.040$. The white finite elements in the void volume fraction contour plots correspond to $f>0.2$.

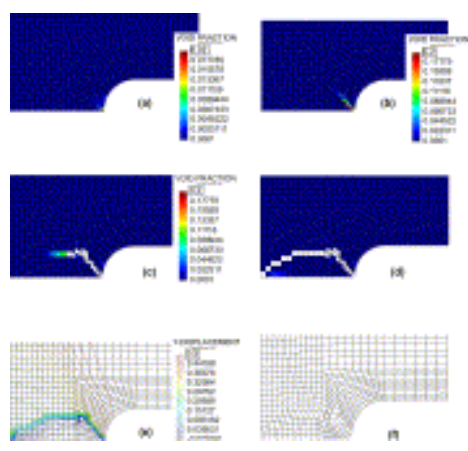

Fig. 10. Field quantity distributions and a deformed mesh showing the evolution of material failure for the $D$-notch geometry with $D / b=0.02$. (a) Void volume fraction distributions at $P / 2 b \sigma_{y}=0.92, \Delta u_{2} / I=0.009$. This coincides with the first time that material instability is detected in the notch root. (b) Void volume fraction distributions at $P / 2 b \sigma_{y}=0.99, \Delta u_{2} / I=0.016$. (c) Void volume fraction distributions at $P / 2 b \sigma_{y}=0.34, \Delta u_{2} / I=0.020$. (d) Void volume fraction distributions at $P / 2 b \sigma_{y}=0.03, \Delta u_{2} / I=0.024$. (e) Distribution of $u_{2}$ at $P / 2 b \sigma_{y}=0.03, \Delta u_{2} / I=0.024$. (f) Deformed mesh at $P / 2 b \sigma_{y}=0.03, \Delta u_{2} / I=0.024$. The white finite elements in the void volume fraction contour plots correspond to $f>0.2$. 
Inclusion of weak discontinuity modes in the numerical procedure results in an increase in the computational cost that can be estimated. The CPU time to solve the case in Fig. 8(b) with $D / b=0.02$ is compared with that for the case without any discontinuity modes under the following conditions:

(i) The solution named without any weak discontinuity modes was obtained using a standard B-bar quadrilateral finite element with four integration points.

(ii) The solution with $D / b=0.02$ was obtained using six quadrature points in all finite elements even in those finite elements where the enhanced modes remain inactive throughout the analysis. The computing time for this calculation could obviously be reduced by only including the additional integration points in elements where they are needed.

(iii) Both calculations were run for 2900 steps at which point a complete loss of load carrying capacity was reached. Also in both calculations one iteration per time step was needed.

The required CPU time for computing the solution, with $D / b=0.02$, was $1074 \mathrm{~s}$. While the CPU time for computing the solution with no weak discontinuity modes was $463 \mathrm{~s}$. Thus, the solution with the enhanced modes required a factor of 2.3 more CPU time.

\section{Concluding remarks}

We have presented a method with a finite thickness embedded weak discontinuity band for analyzing problems involving a complete loss of stress carrying capacity that may be preceded by a localization bifurcation. The particular constitutive relation used is one that models ductile fracture due to progressive cavitation. The post-localization response in the band is governed by the pre-localization constitutive relation so that a unified constitutive framework can give rise to a transition from a weak discontinuity to a strong discontinuity. The band thickness enters the formulation as a material parameter. Its value is independent of the finite element discretization which is in contrast to the cell model of Xia and Shih (1995) where the material length scale and the mesh size are strongly coupled. The numerical examples have illustrated the capabilities of the methodology. In order to focus on the basic numerical issues, we have neglected geometry changes in the formulation presented. In work in progress, the methodology presented here is being extended to a full finite deformation framework and being used to analyze ductile fracture problems. Some implementation details differ but, as in the simpler context considered here, the finite thickness band methodology provides a regularization which permits convergent localization solutions to be obtained that allow for the transition from localization to the creation of new free surface with a hydrostatic stress dependence as needed for modeling ductile fracture. We also emphasize that this methodology can be used with any rate independent constitutive relation that permits a complete loss of stress carrying capacity. 


\section{Acknowledgements}

A.E.H. and P.J.S. are grateful for financial support from ANPCYT of Argentina through grant PICT-2005-34273. J.O. and A.E.H. are pleased to acknowledge support from the Spanish Ministry of Science and Technology through grant BIA2005-09250-C03-03. A.E.H. also express his acknowledgments to Conicet from Argentina and the Fulbright program for supporting his visit to Brown University.

\section{References}

Bai and Wierzbicki, 2008 Y. Bai and T. Wierzbicki, A new model of metal plasticity and fracture with pressure and lode dependence, Int. J. Plasticity 24 (2008), pp. 1071-1096.

G. Barenblatt, Mathematical theory of equilibrium cracks, Adv. Appl. Mech. 7 (1962), pp. 56129.

Becker et al., 1988 R. Becker, A. Needleman, O. Richmond and V. Tvergaard, Void growth and failure in notched bars, J. Mech. Phys. Solids 36 (1988), pp. 317-351.

T. Belytschko, S. Loehnert and J. Song, Multiscale aggregating discontinuities: a method for circumventing loss of material stability, Int. J. Numer. Methods Eng. 73 (2008), pp. 869-894.

Besson et al., 2001 J. Besson, D. Steglich and W. Brocks, Modeling of crack growth in round bar and plane strain specimens, Int. J. Solids Struct. 38 (2001), pp. 8258-8284.

Besson et al., 2003 J. Besson, D. Steglich and W. Brocks, Modeling of plane strain ductile rupture, Int. J. Plasticity 19 (2003), pp. 1517-1541.

Cervera et al., 2001 M. Cervera, C. Agelet and M. Chiumenti, COMET; Multi Purpose Coupled Nonlinear Program for Steady and Transient Conditions, Technical University of Catalonia, Barcelona (2001).

Chu and Needleman, 1980 C. Chu and A. Needleman, Void nucleation effects in biaxially stretched sheets, J. Eng. Mater. Tech. 102 (1980), pp. 249-256.

Dugdale, 1960 D. Dugdale, Yielding of steel sheets containing slits, J. Mech. Phys. Solids 8 (1960), pp. 100-104

Gologanu et al., 1997 M. Gologanu, J.-B. Leblond, G. Perrin and J. Devaux, Recent extensions of Gurson's model for porous ductile metals, continuum micromechanics. In: P. Suquet, Editor, CISM Courses and Lectures, Springer-Verlag, New York (1997), p. 377.

Gurson, 1975 Gurson, A., 1975. Plastic Flow and Fracture Behavior of Ductile Materials Incorporating Void Nucleation, Growth and Interaction. Ph.D. thesis, Brown University.

Hadamard, 1903 Hadamard, J., 1903. Leçons sur la propagation des ondes et les equations de I'hydrodynamique. Librairie Scientifique A. Hermann et Fils, Paris.

Hill, 1962 R. Hill, Acellerations waves in solids, J. Mech. Phys. Solids 10 (1962), pp. 1-16.

A. Hillerborg, M. Modéer and P. Petersson, Analysis of crack formation and crack growth in concrete by means of fracture mechanics and finite elements, Cement Concrete Res. 6 (1976), pp. $773-782$. 
Hughes, 1980 T. Hughes, Generalization of selective integration procedures to anisotropic and non-linear media, Int. J. Numer. Methods Eng. 15 (1980), pp. 1413-1418

Leblond et al., 1994 J. Leblond, G. Perrin and J. Devaux, Bifurcation effects in ductile metals with damage delocalization, J. Appl. Mech. 61 (1994), pp. 236-242.

$\mathrm{Li}$ and Karr, $2009 \mathrm{Y}$. Li and D. Karr, Prediction of ductile fracture in tension by bifurcation, localization, and imperfection analyses, Int. J. Plasticity. 25 (2009), pp. 1128-1153

Linder and Armero, 2007 C. Linder and F. Armero, Finite elements with embedded strong discontinuities for the modeling of failure in solids, Int. J. Numer. Methods Eng. 72 (2007), pp. $1391-1433$

E. Lorentz, J. Besson and V. Cano, Numerical simulation of ductile fracture with the rousselier constitutive law, Comput. Methods Appl. Mech. Eng. 197 (2008), pp. 1965-1982.

Manzoli and Shing, 2006 O. Manzoli and P. Shing, A general technique to embed non-uniform discontinuities into standard solid finite elements, Comput. Struct. 84 (2006), pp. 742-757.

N. Moes, J. Dolbow and T. Belytschko, A finite element method for crack growth without remeshing, Int. J. Numer. Methods Eng. 46 (1999), pp. 131-150.

Needleman, 1987 A. Needleman, A continuum model for void nucleation by inclusion debonding, J. Appl. Mech. 54 (1987), pp. 525-531.

Needleman and Rice, 1978 A. Needleman and J. Rice, Limits to ductility set by plastic flow localization, Mechanics of Sheet Metal Forming, Plenum Press, New York (1978) pp. 237-265.

Needleman and Tvergaard, 1984 A. Needleman and V. Tvergaard, An analysis of ductile rupture in notched bars, J. Mech. Phys. Solids 32 (1984), pp. 461-490.

Needleman et al., 1992 A. Needleman, V. Tvergaard and J. Hutchinson, Void growth in plastic solids, Topics in Fracture and Fatigue, Springer-Verlag, New York (1992) pp. 145-178.

Oliver and Huespe, $2004 \mathrm{~J}$. Oliver and A. Huespe, Theoretical and computational issues in modelling material failure in strong discontinuity scenarios, Comput. Methods Appl. Mech. Eng. 193 (2004), pp. 2987-3014

Oliver et al., 2003 J. Oliver, A. Huespe and E. Samaniego, A study on finite elements for capturing strong discontinuities, Int. J. Numer. Methods Eng. 56 (2003), pp. 2135-2161.

Oliver et al., 2004 J. Oliver, A. Huespe, E. Samaniego and E. Chaves, Continuum approach to the numerical simulation of material failure in concrete, Int. J. Numer. Anal. Methods Geomech. 28 (2004), pp. 609-632.

Oliver et al., 2002 J. Oliver, A.E. Huespe, M.D.G. Pulido and E. Chaves, From continuum mechanics to fracture mechanics: the strong discontinuity approach, Eng. Fract. Mech. 69 (2002), pp. 113-136

Ortiz et al., 1987 M. Ortiz, Y. Leroy and A. Needleman, A finite element method for localized failure analysis, Comput. Methods Appl. Mech. Eng. 61 (1987), pp. 189-214.

Remmers et al., 2003 J. Remmers, R. de Borst and A. Needleman, A cohesive segment method for the simulation of crack growth, Comput. Mech. 31 (2003), pp. 69-77 
Rice, 1968 J. Rice, A path independent integral and the approximate analysis of strain concentration by notches and cracks, J. Appl. Mech. 35 (1968), pp. 379-386.

Rice, 1976 J. Rice, The Localization of Plastic Deformation. In: W. Koiter, Editor, Amsterdam, North-Holland (1976), pp. 207-220.

Saje et al., 1982 M. Saje, J. Pan and A. Needleman, Void nucleation effects on shear localization in porous plastic solids, Int. J. Fract. 19 (1982), pp. 163-182

Sánchez et al., 2008 P. Sánchez, A. Huespe and J. Oliver, On some topics for the numerical simulation of ductile fracture, Int. J. Plasticity 24 (2008), pp. 1008-1038.

Siegmund and Brocks, 2000 T. Siegmund and W. Brocks, A numerical study on the correlation between the work of separation and the dissipation rate in ductile fracture, Eng. Fract. Mech. 67 (2000), pp. 139-154.

Thomas, 1961 T. Thomas, Plastic Flow and Fracture in Solids, Academic Press, New York (1961).

Tvergaard, $1981 \mathrm{~V}$. Tvergaard, Influence of voids on shear band instabilities under plane strain conditions, Int. J. Fract. 17 (1981), pp. 389-407.

Tvergaard, $1982 \mathrm{~V}$. Tvergaard, On localization in ductile materials containing spherical voids, Int. J. Fract. 18 (1982), pp. 237-252. Tvergaard, 1990 V. Tvergaard, Material failure by void growth to coalescence, Adv. Appl. Mech. 27 (1990), pp. 83-151.

Tvergaard and Needleman, 1984 V. Tvergaard and A. Needleman, Analysis of the cup-cone fracture in a round tensile bar, Acta Metall. 32 (1984), pp. 157-169.

Wells and Sluys, 2001 G. Wells and L. Sluys, A new method for modelling cohesive cracks using finite elements, Int. J. Numer. Methods Eng. 50 (2001), pp. 2667-2682.

L. Xia and C. Shih, Ductile crack growth i. a numerical study using computational cells with microstructurally based length scales, J. Mech. Phys. Solids 43 (1995), pp. 233-259.

Xu and Needleman, 1994 X.-P. Xu and A. Needleman, Numerical simulations of fast crack growth in brittle solids, J. Mech. Phys. Solids 42 (1994), pp. 1397-1434. 